\title{
Relaciones sociales y trayectorias biográficas: hacia un enfoque comprensivo de los modos de influencia ${ }^{1}$
}

\author{
Sylvain Bourdon, ERTA - Université de Sherbrooke (Canadá)²
}

\begin{abstract}
Resumen
El objetivo del presente texto es proponer algunos elementos para un enfoque comprensivo de la influencia de las relaciones en las trayectorias biográficas. El análisis se apoya en los datos de un estudio longitudinal sobre el papel que desempeñan la familia y las redes en la perseverancia en los estudios, realizado con 96 jóvenes que inician su trayectoria postsecundaria. En lugar de abordar la influencia en términos de características sociales, o a partir de un enfoque estructural de las redes, proponemos aquí tratarla bajo el aspecto de las relaciones sociales y el sentido que toman éstas para los sujetos. Este enfoque permite examinar de qué manera se imbrican la influencia en la relación y la relación en la influencia, aclarar mejor ciertas dinámicas de las relaciones en un contexto de bifurcación biográfica, y esclarecer el papel que desempeñan las relaciones en la agencia de los individuos. También permite resaltar el papel importante que desempeña la historia compartida en la influencia de las relaciones sobre la trayectoria biográfica.
\end{abstract}

Palabras clave: Redes sociales, influencia, selección, perspectiva biográfica.

\section{Abstract}

This paper analyses the influence of relations in biographic trajectories. It is based in a longitudinal analysis on the role of the family in the studies of 96 youth people after Secondary School. It analyses selection and influence processes, as well as the dynamics of relations in a context of biographical bifurcation. The role of relations and agency is also debated. The study emphasises the role of a shared history in the influence of relations on the biographical trajectory.

Key words: Social Network, Influence, Selection, Life-course.

\footnotetext{
${ }^{1}$ Una versión anterior de este texto fue presentada durante el Congreso de la Association canadienne pour la science (ACFAS) en Québec el 5 de mayo 2008. El autor desea agradecer a Elena Pou del INRS Urbanisation, Culture et Société por la traducción al español, así como a los evaluadores anónimos y a los editores por sus constructivos comentarios.

${ }^{2}$ Enviar correspondencia a: Sylvain Bourdon Sylvain.Bourdon@USherbrooke.ca
} 


\section{REDES- Revista hispana para el análisis de redes sociales \\ Vol.16,\#6, Junio 2009 \\ http: // revista-redes. rediris.es}

\section{I ntroducción}

La cuestión de la influencia de las relaciones sociales en las trayectorias de vida de los individuos, además de su interés teórico, reviste una pertinencia particular para una sociología interpelada por la práctica, particularmente en el marco de apoyo a las poblaciones en estado de precariedad o que son susceptibles de estarlo. Pensamos aquí en las intervenciones en las que, queriendo evitar hacerse cargo de las personas, con los aspectos infantilizantes y los problemas que esto engendra, se busca actuar sobre el tejido social y la toma del poder por parte de las personas. Esta perspectiva es también muy interesante para el estudio de la juventud, pues es una edad de la vida precisamente marcada por una fuerte tensión entre dependencia y autonomía, objeto también de numerosas intervenciones sociales. La juventud ofrece un terreno de estudio particularmente fértil para examinar la influencia, puesto que los jóvenes pueden ser especialmente móviles, tanto desde el punto de vista relacional como social. De hecho, puede pensarse que no es una coincidencia que los jóvenes estén bajo estos dos aspectos a la vez.

El objetivo de este texto es proponer algunos elementos de un enfoque comprensivo de la influencia de las relaciones en las trayectorias biográficas. Entre los análisis de estructuras sociales que plantean los apremios macrosociales que determinan acciones individuales, así como las concepciones fenomenológicas que tienden a desatender el papel de los contextos sociales, esta propuesta viene a complementar los análisis de las relaciones y de las redes sociales que se interesan por los lazos y las interacciones entre individuos. Esta propuesta se inscribe como complemento de los análisis cuantitativos y estructurales de las redes sociales que buscan enriquecer al insistir en el papel que desempeñan los intercambios en las relaciones sociales y en el sentido que toman para los sujetos. Se aborda aquí la influencia como mecanismo relacional, y el enfoque comprensivo permite examinar la manera en que se imbrican la influencia en la relación y la relación en la influencia, y esclarecer mejor ciertas dinámicas de las relaciones en contextos de bifurcación biográfica y aclarar el papel de las relaciones en la agencia de los individuos. También permite resaltar el papel importante que desempeña la historia compartida en la influencia de las relaciones sobre la trayectoria biográfica. 
REDES- Revista hispana para el análisis de redes sociales

Vol.16,\#6, Junio 2009

http: // revista-redes. rediris.es

Las proposiciones de este texto se apoyan en el análisis de los datos de la encuesta Famille, réseaux et persévérance au collégial (Familia, redes y perseverancia en la escuela postsecundaria) (Bourdon, Charbonneau, Cournoyer y Lapostolle, 2007) ${ }^{3}$ que busca documentar las trayectorias de acceso a los estudios postsecundarios en la perspectiva de las redes sociales y la interacción entre los distintos calendarios de acontecimientos (familiar, amoroso, escolar, profesional, residencial) que construyen el paso a la vida adulta. El protocolo de la encuesta y su instrumentación están inspirados en el panel Sociabilité et insertion sociale (sociabilidad e inserción social) organizado en 1995, en Caen, por Claire Bidart, Alain Degenne, Lise Mounier y Daniel Lavenu (Bidart, Mounier, Pellissier, 2002) ${ }^{4}$.

La primera cohorte de la encuesta Famille, réseaux et persévérance au collégial se inició con 96 jóvenes de 17 a 23 años que se inscribieron por primera vez en el otoño 2004 en uno de los tres establecimientos escolares asociados con el proyecto. Estos jóvenes aceptaron participar en un seguimiento longitudinal que consistía en establecer un inventario de su red social y un calendario de acontecimientos, y luego participar en una entrevista semi-estructurada. Para cada ola, esta entrevista permite abordar los vínculos entre los acontecimientos enumerados en el calendario, el sentido y las representaciones asociados con los cambios acontecidos entre los periodos y con las elecciones y sus impactos, las transformaciones de la red, la evolución de la calidad de las relaciones dentro de la red, las cuestiones de negociación en torno al acceso a ciertos recursos, la satisfacción general en relación con la trayectoria escolar, los demás acontecimientos del calendario y las relaciones en la red social. Los datos que aquí se presentan están sacados de las tres primeras olas de encuesta realizadas al comienzo de las clases del otoño $2004(\mathrm{~N}=96)$, en la primavera del $2005(\mathrm{~N}=86)$ y a fines del otoño $2005 \mathrm{~N}=84$ ).

La encuesta utilizada nos llevará a interesarnos principalmente por las bifurcaciones escolares de los jóvenes, pero las proposiciones de comprensión que se desprenden pueden esclarecer los procesos de influencia que se desarrollan en sujetos y en contextos mucho más diversificados.

\footnotetext{
${ }^{3}$ Los datos provienen de un proyecto de investigación financiado en el marco del programa Action concertée Persévérance et réussite scolaire del Fonds québécois de recherche sur la société et la culture (FQRSC) financiado por el ministerio de Educación, Ocio y Deporte de Québec (MELS, por sus siglas en francés).

${ }^{4}$ Se encuentra una comparación de las dos encuestas en Bidart, Bourdon y Charbonneau (2007).
} 
REDES- Revista hispana para el análisis de redes sociales

Vol.16,\#6, Junio 2009

http: // revista-redes. rediris.es

\section{Biografía e influencia}

La influencia de lo social en las trayectorias de vida puede ponerse en tensión con el potencial de agencia de los individuos, o sea su capacidad de influir en el curso de su existencia, ante los determinismos y los efectos de coyuntura. La perspectiva biográfica (Life course) considera que los individuos están implicados en secuencias de acontecimientos cronológicamente normativos, en entornos y papeles sociales estructurados por instituciones sociales. Permite examinar la capacidad de los individuos de influir en el curso de su vida, individualizar sus trayectorias al eximirlas de las normas y rituales establecidos, frente al riesgo y la incertidumbre (Rudd y Evans, 1998; Wynn y Dwyer, 1999). Según este enfoque, las transiciones vividas por los individuos están siempre inscritas en trayectorias que les dan una forma y un sentido distintivos. Uno de los principios del enfoque biográfico enunciado por Elder (1998) es que las vidas son interdependientes y que las influencias sociales e históricas son expresadas y percibidas a través de redes de relaciones compartidas (Charbonneau, 2005).

Varios trabajos empíricos han puesto en evidencia la influencia de las redes sociales en los individuos y los colectivos. La psicología social se ha interesado por los mecanismos de formación de la opinión, de difusión de los rumores, o de las normas en los grupos. Por ejemplo, Rojas y Howe (2004) aislaron el efecto de la estructura de la red social en la influencia que puede tener un individuo dentro de un grupo en el momento de la formación de una opinión. Otros han resaltado el papel importante que desempeñan las redes en la difusión de la innovación (Grossetti y Bès, 2001). También han podido observarse numerosas asociaciones entre la estructura de las redes y los comportamientos, como en los trabajos recientes de Christakis y Fowler (2007; 2008) sobre la influencia de la red en la propagación de la obesidad y del tabaquismo. Pese a que se ponen en evidencia las asociaciones, aún se comprende mal de qué manera juega, al nivel de los mecanismos de interacción y del sentido que dan los individuos a las relaciones, la influencia de las redes en las trayectorias de vida, y particularmente las interacciones entre los procesos de selección y de influencia que se imbrican en la evolución de las redes de relaciones y las trayectorias de vida.

Pero entonces, ¿qué es la influencia? El diccionario general Robert nos da algunas definiciones interesantes. Primero, la influencia es un "Flujo proveniente de los astros que actúa sobre los hombres y las cosas". También puede ser una "Acción ejercida por 
REDES- Revista hispana para el análisis de redes sociales

Vol.16,\#6, Junio 2009

http: // revista-redes. rediris.es

una cosa, una situación, sobre alguien o algo", especificándose que dicha acción, cuando es ejercida por una persona, puede ser voluntaria o no. Esta noción de flujo es aquí interesante, particularmente por su imprecisión y el carácter algo esotérico que puede connotar. Se piensa en una corriente, un fluido, una "energía", pero sin tener una idea precisa de lo que se transmite, ni cómo, ni por qué. Los trabajos que mencionan asociaciones estadísticas entre la estructura de las redes sociales y los comportamientos o valores de los individuos, aún cuando son esclarecedores, no permiten llegar a una comprensión de la influencia como fuente de sentido para los sujetos. Resaltan las estructuras, a veces los intercambios, pero hacen poco caso de la perspectiva que tienen los sujetos sobre la articulación entre el manejo de sus vidas y sus relaciones.

\section{Figuras de la influencia}

Interesarse por la influencia en el marco de las trayectorias de vida significa interesarse por los ingredientes de la acción. Michel Grossetti (2004) nos ofrece una clasificación muy interesante de los recursos cognitivos como ingredientes de la acción, en cinco categorías: las finalidades, los valores, las rutinas, los afectos y las teorías. Expone que "Las finalidades se presentan como uno de los ingredientes más flexibles o inestables de la acción, uno de los más sensibles a las contingencias. Es también uno de los aspectos importantes de la libertad de acción para unos, y de las tentativas de control para otros." (p. 86). Así, no es sorprendente encontrar las finalidades, a las que también puede atribuirse el concepto de proyecto (Bidart y Lavenu, 2001; Boutinet, 2005), en el fundamento de un enfoque comprensivo de la influencia.

Si se examina la influencia desde el punto de vista de las finalidades de los actores presentes, se imponen tres figuras que marcan igual número de temporalidades de la influencia. La primera figura consiste, para un sujeto, en estar inscrito en la historia de otro. Esta inscripción puede toparse o no con resistencias, como en el caso del joven para quien representa un peso el proyecto (escolar, profesional, familiar...) de sus padres, de su familia. Es el caso de Aurélie, la primera de su familia en realizar estudios postsecundarios y que siente de manera particularmente aguda la misión de pionera que le ha confiado la familia, en calidad de estudiante de primera generación ${ }^{5}$.

\footnotetext{
${ }^{5}$ Ver Pascarella et al. (2004) para una revista sobre el concepto de estudiante de primera generación.
} 
REDES- Revista hispana para el análisis de redes sociales

Vol.16,\#6, Junio 2009

http: // revista-redes. rediris.es

Aurélie - Sabes, soy la primera de la familia, y todo eso. [...] Sabes, me siento presionada. Desde que soy pequeña, quiero ser buena alumna. Quiero ser esto, quiero ser aquello, me digo que tengo que ser así porque si no, no van a sentirse orgullosos, ya sabes. Y pues, ya sabes, seguro que voy a ir a la universidad, garantizado. No voy a dejar la escuela, seguro. [...] Sólo que, ya sabes, a lo mejor perdí la ocasión de sacar un diploma técnico, o un diploma de estudios profesionales (DEP) porque me decía "Tengo que ir a la universidad porque, ya sabes, ellos quieren que vaya".

La segunda figura es en parte el espejo de la primera: la inscripción de otro en su propio proyecto, en su propia historia. Es el caso de los hijos u otros familiares dependientes, pero también de las relaciones amorosas y de varias relaciones de amistad fuertes (Ferrand, 2006). A este respecto, el ejemplo de Marjolaine es elocuente. Hija de padres que no estudiaron a nivel postsecundario, inicia sus estudios después de haber obtenido resultados escolares más bien bajos en la secundaria. Durante la primera entrevista, dice que su elección de estudios está muy influenciada por sus amigos y su pareja Julien, quien sigue el mismo programa que ella, pero que no se ocupa mucho de sus estudios. Cuando se la vuelve a ver la primavera siguiente, está saliendo con Joël, un estudiante universitario. A todo lo largo de la entrevista, puede observarse que la trayectoria de su nuevo enamorado ejerce mucha influencia en sus elecciones, y que Marjolaine le atribuye una parte importante del control sobre su propia trayectoria.

Marjolaine - [P: ¿Y tus estudios?] Bueno, espero continuar, en el fondo. [¿Existe el riesgo que cambies o abandones? Existen acontecimientos que podrían causar esto?] No creo, en todo caso no por el momento. A menos que mi novio... Bueno, en el fondo, si algo le pasa y... Digamos que tenemos que mudarnos a otra parte, o... Yo lo seguiría, eso seguro. O sea que eso sería. Creo que sería la única cosa que podría causar eso.

Marjolaine "espera" proseguir, si la trayectoria de Joël le da la oportunidad. Puede pensarse que si hubiera seguido con Julien, menos estudioso que Joël, su trayectoria postsecundaria hubiera corrido más riesgo de verse interrumpida. Por cierto que durante nuestro tercer encuentro, mientras persevera tanto en sus estudios postsecundarios como en su relación con Joël, menciona que pensó en algunas 
REDES- Revista hispana para el análisis de redes sociales

Vol.16,\#6, Junio 2009

http: // revista-redes. rediris.es

ocasiones en interrumpir sus estudios, o por lo menos en cambiar de programa, y cuenta mucho con el apoyo de los demás para ayudarla a proseguir.

Marjolaine - Seguro que hablo a menudo de esto con mis padres, con mi compañero, y todo, para saber, para que me ayuden. Porque sola, a veces es duro. Y pues abandonar, a veces me hago boba "Ah, yo dejo la escuela. ¡Ya estoy harta!". Pero sabes, no es nunca verdaderamente... Sabes, no lo haría ahora, creo, a menos que algo sucediera. Pero si no, no lo haría.

Estas dos primeras figuras, la inscripción en la historia del otro, y la inscripción del otro en su propia historia, se sitúan en una temporalidad que hace puente entre el pasado y el futuro, elaborando una historia común, ya bien establecida o en devenir, por ejemplo en el caso de los hijos por nacer o las relaciones fuertes nacientes.

La importancia de la historia compartida en estas relaciones es primordial. Crea similitud allí donde sin embargo pueden persistir diferencias. Crea también afecto, fuente de compromiso recíproco, aspecto fundamental de toda relación social (Finch y Mason, 1993; Grossetti, 2008). Así, en la biografía se imbrica la historia, y en el hecho de compartir se entabla, se elabora y se confirma la relación.

Opuestamente, la tercera figura está anclada en el presente, incluso en el instante. Es la de la relación de paso, por definición escasamente inscrita en la historia pero susceptible de abrir puertas hacia nuevos horizontes y extender el repertorio de los posibles. Por ejemplo, fue posible identificar un caso particular de esta figura que varios jóvenes nombran como sus "amigos de escuela", esos "pares", frecuentados en el marco escolar, y que se pierden de vista, sin pesar ni alegría, al cambiar de escuela o al abandonarla. Puesto que tienen historias diferentes, cada cual es susceptible de abrir su "pequeño mundo" (Bidart y Lavenu, 2004), hecho de sus propios conocimientos, experiencias, ideas y relaciones. Por su diversidad de origen, de experiencias y de intereses, y en virtud de la fugacidad potencial de este tipo de relación, ofrecen la ocasión de explorar, sin comprometerse demasiado, universos múltiples y, de allí, profundizar su trayecto de construcción identitaria.

La relación de paso es también la relación "funcional" que puede establecerse entre los interventores y ciertas poblaciones. En el caso de los estudiantes de postsecundaria, puede tratarse del personal docente quien, en un momento de cuestionamiento, o frente a un problema específico, podrá implicarse durante cierto tiempo en un 
REDES- Revista hispana para el análisis de redes sociales

Vol.16,\#6, Junio 2009

http: // revista-redes. rediris.es

intercambio susceptible de tener un efecto importante en la trayectoria del joven. Mientras que en la mayoría de los casos estas relaciones permanecen al límite de lo personal y no suscitan un real compromiso, pudo observarse, en el marco de otras encuestas con poblaciones vulnerables (Charbonneau, 2003), que ciertos sujetos que viven en condiciones de precariedad y aislamiento social importante tienden a integrar a los interventores en su historia y sus redes de relaciones significativas.

Los análisis realizados sobre la evolución del proyecto profesional de los jóvenes en el marco de nuestra encuesta (Cournoyer, 2008) muestran que las relaciones que están más imbricadas en la historia de los sujetos son típicamente fuentes de una influencia que podemos calificar de "convergente". Refuerzan un proyecto ya presente, y son fuentes de seguridad y sostén afectivo. Por su parte, las relaciones menos cargadas de historia tienen más una influencia divergente, y eventualmente desestabilizadora para los jóvenes pues desafían sus concepciones y las abren hacia otros futuros posibles.

\section{Cambios y relaciones}

Como lo recuerdan Steglich, Snijder y Pearson (2007), la observación según la cual los individuos que se agrupan tienden a compartir las mismas características, ya sea en términos de comportamientos, actitudes o valores, ha dado lugar a interpretaciones teóricas divergentes. Por una parte, funciona un mecanismo de selección según el cual la similitud, a través de las afinidades electivas, favorece la construcción y el mantenimiento del vínculo según el antiguo dicho "cada oveja con su pareja" (McPherson y Smith-Lovin, 2001). En el mismo orden de ideas, Kalish y Robins (2006) hicieron el vínculo entre las características de conjunto (cierre y agujeros estructurales) de las redes egocéntricas y ciertas predisposiciones psicológicas de los sujetos de su encuesta. Asimismo, puede observarse cómo funcionan mecanismos de influencia mediante los cuales se difunden las posiciones del grupo entre sus miembros (Friedkin y Johnsen, 1999; Friedkin, 2001). Es el caso de los modelos psicosociológicos particularmente, con los que se busca determinar de qué manera se forma la opinión en grupos pequeños pero que dejan a un lado la cuestión de la formación de los grupos en sí.

Sin embargo, se acepta cada vez más el hecho que los procesos de influencia y de selección están entrelazados en la trayectoria de los individuos. Por ejemplo, Giordano (2003) recuerda, con otros, el "good girl friend effect" sobre los criminales, e invita a realizar investigaciones más a fondo con el fin de lograr distinguir cuál arrastra al otro, 
REDES- Revista hispana para el análisis de redes sociales

Vol.16,\#6, Junio 2009

http: // revista-redes. rediris.es

el cambio de comportamiento o la relación. Muy pocas proposiciones, como la de Steglich et al. (2007), intentan aclarar, al nivel estadístico, el desorden de la selección y de la influencia en la evolución de las redes sociales. Los resultados obtenidos hasta ahora con estos modelos complejos confirman la idea que la selección y la influencia están de hecho estrechamente interrelacionadas y que, aunque es posible distinguirlas parcialmente, la explicación de una parte importante de las variaciones observadas sigue siendo vaga. Además, estos modelos, como los demás modelos estructurales, se ocupan poco de la dimensión socialmente construida del vínculo social que da su sentido, y por ende ciertas características particulares en términos de afecto y estabilidad, a ciertas relaciones. En las sociedades occidentales, los lazos de parentesco distinguen una clase de relaciones que no tienen las mismas características que las demás desde el punto de vista de los procesos de selección. El costo asociado con la ruptura de una relación entre un sujeto y uno de sus padres es muy diferente del que se puede atribuir a la mayor parte de las rupturas amistosas. Es la misma situación para ciertas relaciones contractuales, como el matrimonio o la adopción de niños, que sostienen una forma de perennidad que no tienen en cuenta los modelos estrictamente reticulares. Los análisis de redes sociales, a menudo, hacen poco caso de la parte del poder asociado con ciertas relaciones, y las asimetrías que puede generar al nivel de la obligación. A este respecto, pensamos en las relaciones entre patrón y empleados, o entre interventores y beneficiarios de servicios públicos.

Más allá de esta distinción entre selección e influencia, y teniendo en cuenta su estrecha imbricación, nos parece interesante comprender, en lugar de intentar determinar en que ámbito, conductista o relacional, se inicia la bifurcación (Grossetti, 2004), de qué manera ambos se imbrican y cómo comienza a girar la rueda del cambio, en un sentido o en otro, cómo disminuye o se acelera su velocidad. El estudio de los modos de influencia se sobrepone entonces con el de los modos de entrada y salida de relaciones, de los accesos y distanciamientos en la historia del sujeto; en resumen, de los puntos de inflexión en la trayectoria biográfica y relacional.

\section{Los puntos de inflexión}

Para comprender los puntos de inflexión, puede utilizarse el concepto musical de disonancia, que significa "la discordancia de un conjunto de sonidos que produce una impresión de inestabilidad y tensión, y requiere una resolución". Friedkin (2001), quien estudia la formación de la norma en las redes, recuerda que ésta sirve particularmente 
REDES- Revista hispana para el análisis de redes sociales

Vol.16,\#6, Junio 2009

http: // revista-redes. rediris.es

para reducir la incertidumbre y el conflicto en las interacciones sociales mediante una modificación de la actitud o de la opinión. Así, puede considerarse que la disonancia en una relación es tanto más susceptible de suscitar tensiones cuanto afecta a la historia compartida de los sujetos, considerando el papel importante que desempeña esta última en la existencia misma de la relación. A la inversa, en el caso de las relaciones sin historia, es más bien su contrario, la consonancia, la que, con el tiempo, puede favorecer los acercamientos. Cuando se instala una dualidad en una historia compartida, la disonancia requiere forzosamente una resolución.

Una parte importante de las interacciones para manejar esta disonancia está fundada en discusiones e intercambios de opiniones entre los protagonistas. De estas interacciones en torno a las posibilidades de bifurcaciones en la vida de los sujetos pueden surgir cambios de actitudes o de proyectos, tanto para ego como para alter. Se encuentran así tres maneras principales de manejar la disonancia con un allegado. Consisten en acercarse al otro (converger), acercar al otro hacia sí (hacer converger) o alejarse del otro (modificar la relación). El ejemplo de la relación con los padres cuando surge la posibilidad de interrumpir los estudios ilustra bien esta dinámica. Emmanuelle relata cómo se efectuó la negociación con su madre en torno a su proyecto de interrupción de estudios. La problemática en su caso es importante puesto que le proporcionan casa y comida, arreglo fundamentalmente tributario de la continuidad de su escolarización.

Emmanuelle - [P: Cuando le dijiste a tu madre que querías dejar la escuela por un tiempo, ¿te apoyó?] Al principio no, no quería. No quería para nada porque tenía miedo que ya no volviera yo a la escuela postsecundaria. Porque había dicho que quería soltar la escuela y había comenzado a trabajar y todo eso. Entonces me dijo: "Bueno, sabes, no pienso que sería lo mejor. Va a ser une etapa de tu vida. Vas a haber aprendido durante ese tiempo". Entonces fue que le dije: "Mamá, no necesito tener ceros en todas las materias para saber que las cosas no van bien". Tuvimos una fiesta el fin de semana, y entonces vio que no funcionaba. Que yo no estaba como de costumbre, y entonces me dijo: "Deja de estresarte con eso. Está bien si dejaste el colegio. Lo dejaste". [.... ] Ahora, tengo todo el apoyo de mis padres. De verdad, me apoyan, y dí a entender que no quiero abandonar, no es un abandono escolar. Para nada. Es porque no estoy bien. 


\title{
REDES- Revista hispana para el análisis de redes sociales Vol. 16,\#6, Junio 2009 \\ http: // revista-redes. rediris.es
}

\begin{abstract}
Puede observarse cómo la negociación de sentidos entre Emmanuelle y sus padres sobre lo que en un principio se consideraba como un abandono de su escolaridad, transformó el proyecto en simple interrupción, obteniendo el apoyo de su madre a su proyecto y resolviendo al mismo tiempo la disonancia entre sus versiones de la historia de Emmanuelle. Por lo tanto, la dinámica de influencia no puede comprenderse en sentido único. Las transacciones en torno a los proyectos de vida, a la interconexión de las historias individuales, pueden resolverse tanto mediante una modificación del proyecto de ego como por la reificación de esta bifurcación a los ojos de alter. La imposibilidad de hacerlo podrá generar sufrimiento o un alejamiento, incluso una ruptura en la relación. Numerosos cambios en las redes se producen en consecuencia - o incluso en la anticipación- de bifurcaciones. El caso de Véronique es interesante para ilustrar estas distintas interacciones.
\end{abstract}

\section{Véronique}

Véronique proviene de un medio modesto, sus padres están ambos presentes en su red, sin calificárseles de íntimos (Figura 1).

Con ocasión de su entrada en la escuela postsecundaria, su red cuenta con veintidós miembros, entre ellos tres personas importantes, y cinco de sus amigos han abandonado los estudios, la mayoría de las veces por pequeños empleos, lo que Véronique lamenta es:

Yo no tengo verdaderamente muchos amigos en la escuela postsecundaria, pero sí tengo algunos que piensan ir, ya es algo.

En la primavera siguiente, en la oleada 2, se observa que su red, que incluso desde el principio no era muy extensa, se ha encogido a quince miembros ${ }^{6}$. Habla entonces de hacer la limpieza en sus relaciones para concentrarse mejor en sus estudios y su empleo a medio tiempo, que acaparan cada vez más su tiempo.

Bueno, hice la limpieza. [...] Me siento bien. Estoy contenta porque hay menos gente con quien comparto cosas. Era mucha gente que atender yo sola. Estoy contenta porque me daba cuenta que a veces me sentía rodeada por demasiada gente para lo que podía dar. Ahora, pues tengo menos personas...

\footnotetext{
${ }^{6}$ El número promedio de alters para este grupo de jóvenes es de 30 .
} 
REDES- Revista hispana para el análisis de redes sociales

Vol. 16,\#6, Junio 2009

http:// revista-redes. rediris.es

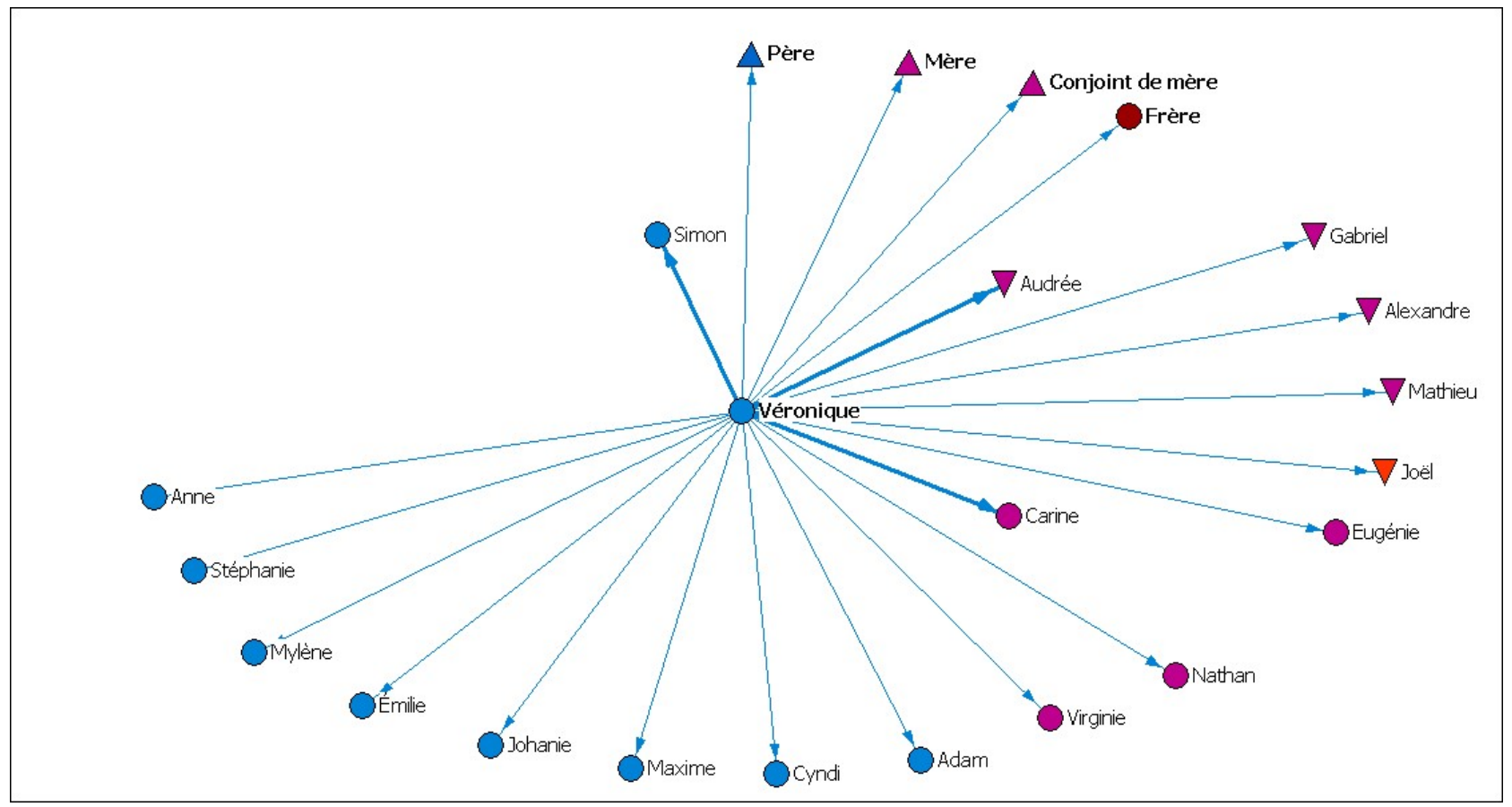

$\begin{array}{ll}\square \square \text { scolarité collégiale et }+ & \bigcirc \text { École } \\ & \triangle_{\text {Scolarité secondaire }}^{\text {Emploi }} \\ & \nabla \text { Petit boulot }\end{array}$

Escolaridad postsecundaria y $+\quad$ Escuela Escolaridad secundaria Empleo

Trabajo pequeño

Figura 1. Padre, Madre, Cónyuge de la madre, hermano.

Véronique estrecha así su red para concentrarse en sus relaciones íntimas. Entre las personas que desaparecen, todas tienen de 17 a 20 años, y varias de ellas interrumpieron sus estudios después de la secundaria (Figura 2).

Pese a ello, su implicación escolar sigue estando en equilibrio frágil con el mantenimiento de vínculos que para ella son importantes. Siente que el tiempo dedicado a la sociabilidad es como una punción en el tiempo de estudio.

Veo más a mis amigos cercanos que antes, y los demás, pues se largaron. En el fondo es eso nada más. [...] A veces, no tengo necesariamente tiempo para verlos, pero como son mis amigos cercanos, tengo ganas de verlos, tengo ganas de hablar con ellos. A lo mejor por eso pierdo un poco de tiempo en mis trabajos, o me retraso. 
REDES- Revista hispana para el análisis de redes sociales

Vol. 16,\#6, Junio 2009

http: // revista-redes. rediris.es

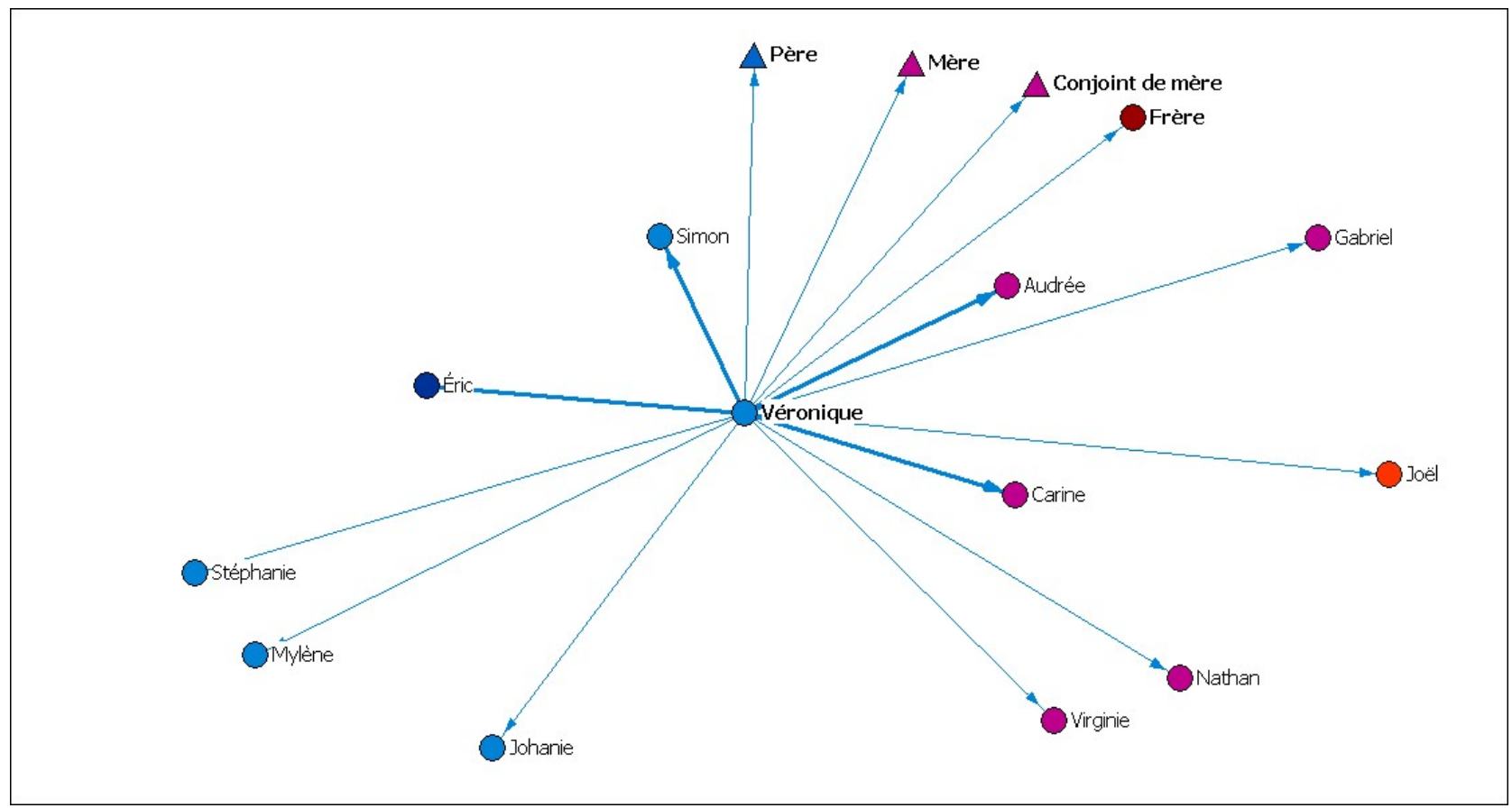

\begin{tabular}{|ll}
$\square \square$ scolarité collégiale et + & Ó́cole \\
$\square \square$ scolarité secondaire & $\triangle$ Emploi \\
& $\nabla$ Petit boulot
\end{tabular}

Figura 2. Relaciones íntimas.

Esta "poda" que ejerce en su red de amigos no funciona de la misma manera con la familia. Sus padres no viven juntos desde hace tiempo. Su madre vive con un nuevo cónyuge, y ambos tienen una escolaridad de nivel secundaria. Según Véronique, su madre apoya muy poco sus esfuerzos escolares, lo que las coloca en fuerte disonancia en relación con su situación actual y sus proyectos. Su madre no comprende su situación de estudiante, los esfuerzos que debe realizar, la importancia que otorga al trabajo escolar; tampoco comprende ni puede aportarle las condiciones necesarias para su éxito.

Por esta razón, desde el principio de sus estudios postsecundarios, elige residir únicamente en casa de su padre, después de haber vivido en custodia alternada durante varios años. Sin embargo, hasta el tercer encuentro, dice que trata de que su madre se interese por sus estudios, y que intenta un acercamiento, pero sin lograrlo realmente. Todo lo más, nota que su madre siente cierto orgullo cuando se convence 


\section{REDES- Revista hispana para el análisis de redes sociales Vol. 16,\#6, Junio 2009 \\ http: // revista-redes. rediris.es}

de que Véronique piensa dedicarse a los estudios universitarios. Véronique rechaza entonces este aval de su madre, que le parece demasiado superficial y tardío.

Como ahora, ya pronto acabo mi diploma de estudios postsecundarios, y me dice, y le dice a todo el mundo "Ah, es gracias a mí" y todo eso. Pero no es gracias a ella. Nunca me animó, pero se diría que se da todo el crédito una vez que está hecho. No cree en mí, que puedo hacerlo, pero cuando lo he hecho no le queda otro remedio que decir "Ah, bien, es cierto, eres capaz"... Siempre es así con mi madre.

La actitud de su padre parece ser diferente. Aunque era distante y se implicaba mínimamente en el apoyo escolar de Véronique cuando entró a la escuela postsecundaria, ahora la apoya cada vez más, al punto de haberle ofrecido instalarle un apartamento en el sótano de su casa, donde ella sigue viviendo en el momento de nuestro tercer encuentro, para apoyar su ingreso a la universidad.

En la oleada 3, todos los amigos y conocidos mencionados en su red, que cuenta ahora dieciséis miembros, estudian, con la excepción de Joël, un amigo de infancia quien dejó la escuela sin diploma de secundaria y al que ve con muy poca frecuencia (Figura 3). Ha conservado una relación privilegiada con Simon, quien fue su enamorado por más de tres años en la oleada 1 y con quien cortó durante su primer año de escuela postsecundaria; comparten objetivos escolares comunes que ella considera sumamente inspiradores. Nos lo dirá en la oleada 3:

A él lo veo un poco como un modelo, en el sentido que tiene un año más que yo, estudia Derecho, lo que se acerca quizás un poco de lo que yo quiero hacer. $\mathrm{O}$ sea que es seguro que... yo veo que tenemos intereses comunes respecto a los estudios.

Véronique parece también haber logrado forjarse un entorno social que corresponde a sus ambiciones escolares.

Bueno, tengo amigos que están en la universidad también, y pienso que encontrarme con esa gente me motiva para ir, porque me siento bien con ellos. Son todos amigos. [...] Si pudiera, estudiaría hasta el fin de mi vida. Pero pienso que es realmente porque no quiero reproducir lo que hicieron mis padres. Mi padre menos, pero mi madre tiene un quinto de secundaria y tiene muchas 
REDES- Revista hispana para el análisis de redes sociales

Vol. 16,\#6, Junio 2009

http: // revista-redes. rediris.es

dificultades en la vida. Y no quiero reproducir eso. Es verdaderamente lo que me motiva para ir a la escuela. Y también la gente que me rodea, mis amigos y todo, forma parte de su camino en la vida. O sea que para mí, es como impensable no ir.

Este círculo de gente que la rodea fue compuesto en ruptura con la herencia parental, materna sobre todo, y en reacción contra ésta. Pero también fue compuesto como precursor de un proyecto de escolarización que se fue confirmando, afirmando y consolidando poco a poco, al punto de volverse, para ella, ineluctable, cuando originalmente no parecía probable.

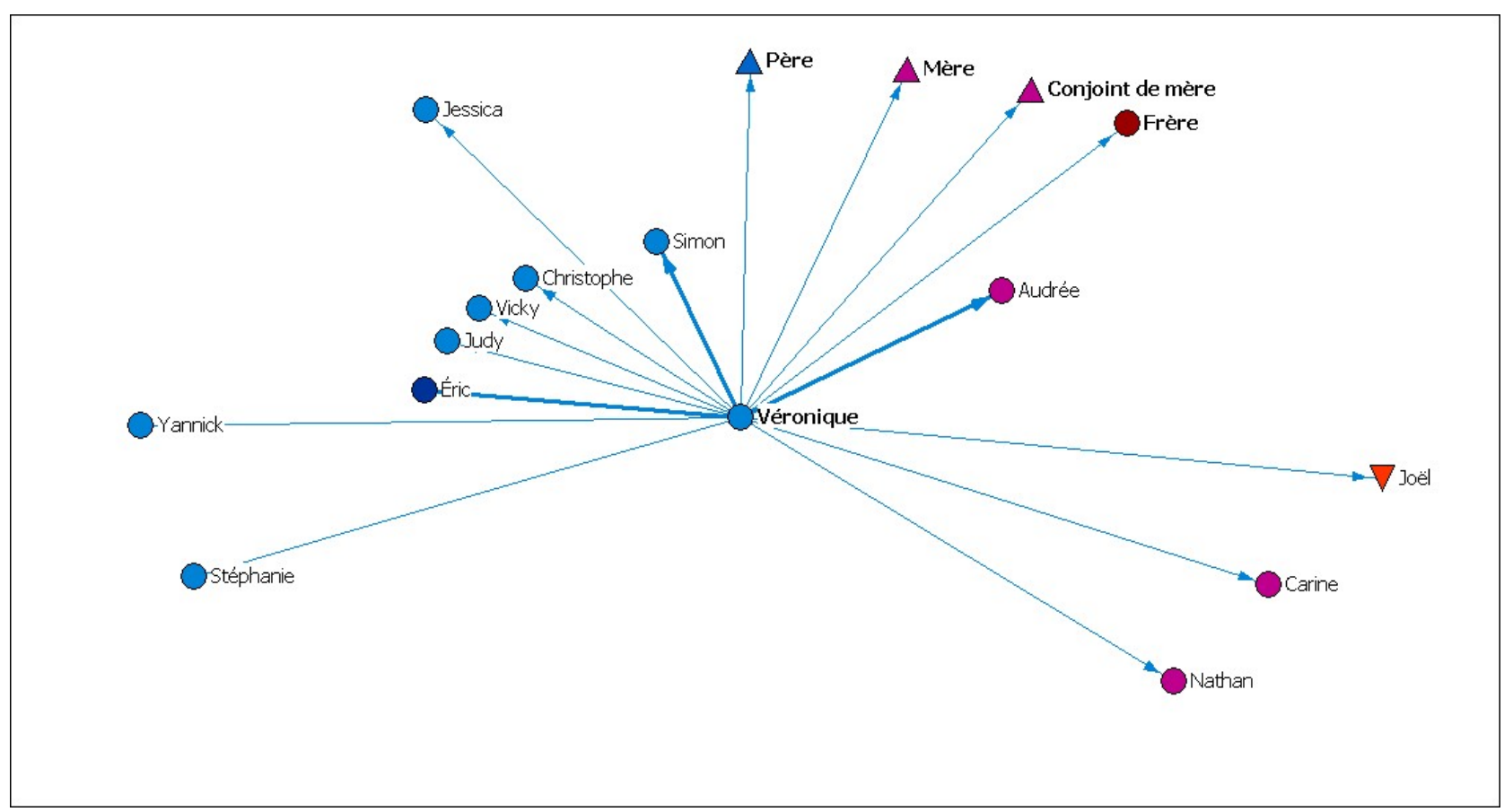

Scolarité collégiale et +

Figura 3. Oleada 3.

\section{Conclusión}

Bidart y Lavenu (2005) habían ya observado que el final de los estudios es un factor importante de disminución del tamaño de las redes y que, a la inversa, la continuación de los estudios puede aumentar su tamaño. Puede observarse aquí de qué manera se registran los cambios biográficos en la composición de la red, y cómo esta composición 
REDES- Revista hispana para el análisis de redes sociales

Vol.16,\#6, Junio 2009

http: // revista-redes. rediris.es

influye a su vez en las transiciones ulteriores, por las posibilidades de vínculo, de intercambio, de acceso a los recursos que éstas permiten o no. Así, la influencia del entorno relacional sobre la trayectoria biográfica se combina con la influencia de la trayectoria sobre este entorno, en una dinámica de reforzamiento o de inflexión de las trayectorias. La acción que ejercen los sujetos en la composición de sus redes se revela como un mecanismo importante, constitutivo de reversibilidad (Charbonneau, 2004) en la trayectoria escolar de los jóvenes adultos que participaron en nuestra encuesta, y susceptible de encontrarse en el centro de varias otras bifurcaciones en las trayectorias de vida.

La mayoría de los análisis de redes sociales que buscan explicar los procesos de selección y de influencia plantean la afinidad entre personas con características similares como el elemento que favorece su encuentro y el reforzamiento del vínculo. Sin embargo, desatienden la parte de selección de las relaciones que pueden efectuarse, no en función de las afinidades actuales, sino anticipando y sosteniendo las modificaciones de la trayectoria de vida, del proyecto, de la historia que tiene en mente el sujeto. Bidart y Degenne (2005) recuerdan que las redes personales tienen una historia, y que están fundadas en historias relacionales. Mientras que son frecuentes las menciones de la importancia de las relaciones en los trabajos que tratan de las historias de vida y los análisis biográficos, siguen siendo escasas cuando se trata de movilizar los conceptos y herramientas provenientes del análisis de las redes sociales, o bien interesarse directamente por la cuestión de su influencia. Los enfoques estructurales de las redes pueden esclarecer las interacciones entre las estructuras de relaciones y ciertos comportamientos o actitudes que guían las trayectorias biográficas. El enfoque comprensivo completa este esclarecimiento, y puede ayudar a establecer puentes entre ambos campos (análisis de las redes sociales y biográficas) y epistemologías (cuantitativa y cualitativa) al ofrecer claves para captar la importancia del sentido que se da a las relaciones en el marco de las trayectorias, y a la negociación del sentido de las bifurcaciones de trayectoria en el marco de las relaciones. El concepto de historia compartida entre el sujeto y las personas con las que está en relación, y de las disonancias que podrían inmiscuirse entre estas historias en el marco de bifurcaciones biográficas, permite resaltar el hecho de que las relaciones no son todas intercambiables, que ocupan lugares distintos en la historia, pasada y por venir, de los sujetos, y que estas diferencias matizarán a su vez los diferentes modos de resolución de las disonancias en la dualidad. También resalta el 
REDES- Revista hispana para el análisis de redes sociales

Vol.16,\#6, Junio 2009

http: // revista-redes. rediris.es

papel que desempeñan las anticipaciones, tanto en los recorridos como en la transformación de las relaciones, particularmente por el fenómeno de "limpieza" operado en el marco de ciertas bifurcaciones.

\section{Referencias}

Bidart, Claire, Bourdon, Sylvain y J ohanne Charbonneau (2007). Le rapport au travail de jeunes au Québec et en France: mise en perspective longitudinale, Coloquio Approches longitudinales: confrontations franco-canadiennes, EHEES, París. [http://www.cmh.ens.fr/hoparticle.php?id_art=13]

Bidart, Claire y Alain Degenne (2005). "Introduction: the dynamics of personal networks", Social Networks, 27(4), 283-187.

Bidart, Claire y Daniel Lavenu (2001). Projets et trajets, contraintes et contingences : qu'est-ce qui fait bifurquer des parcours ? Documento de trabajo. Huitièmes Journées de Sociologie du Travail. Marchés du travail et différentiations sociales. Approches comparatives. Aix-en-Provence - 21-22-23 junio 2001. [http://www.univaix.fr/lest/lesdocuments/lesdocumentsdetravail/bidart/projtrajet.pdf]

Bidart, Claire, Mounier, Lise y Anne Pellissier (2002). La construction de I'insertion socio-professionnelle des jeunes à l'épreuve du temps, une enquête longitudinale. Rapport final, Aix-en-Provence/París: CNRS-MRSH.

Bourdon, Sylvain, Charbonneau, Johanne, Cournoyer, Louis y Lynn Lapostolle (2007). Famille, réseaux et persévérance au collégial Phase 1. Rapport de recherche. Sherbrooke: Équipe de recherche sur les transitions et l'apprentissage (ÉRTA).

Boutinet, Jean-Pierre (2005). Anthropologie du projet, Paris : Presses universitaires de France.

Charbonneau, J ohanne (2005). "La question des temporalités dans I'analyse du social", en Daniel Mercure (dir.), L'analyse du social, Québec: Presses de I'Université Laval, pp. 169-182.

Charbonneau, J ohanne (2003). Adolescentes et mères. Histoires de maternité précoce et soutien du réseau social, Québec: Presses de l'Université Laval.

Christakis, Nicholas A., y James H. Fowler (2008). "The collective dynamics of smoking in large social networks". New England journal of medicine, 358, 2249-2258. 
REDES- Revista hispana para el análisis de redes sociales

Vol.16,\#6, Junio 2009

http: // revista-redes. rediris.es

Christakis, Nicholas A., y James H. Fowler (2007). "The spread of obesity in a large social network over 32 years", New England journal of medicine, 357, 370-379.

Cournoyer, Louis (2008). L'évolution des projets professionnels de colégiennes et collégiens lors des 18 premiers mois d'études collégiales: le rôle des relations sociales, Tesis de doctorado inédita, Université de Sherbrooke.

Felmlee, Diane H. (2003). "Interaction in social networks". In Delamater, J. (Dir.) Handbook of social psychology (pp. 389-409), New-York: Kluwer/Plenum.

Ferrand, Alexis (2006). "Redes de discusión heterogéneas y pluralismo cognitivo". Redes. Revista Hispana para el Análisis de Redes Sociales, 10 (2). Consulta [1-052008] en http://revista-redes.rediris.es.

Finch, Janet V. y Jennifer Mason (1993). Negociating family responsibilities, London: Routledge.

Friedkin, Noah E. (2001). "Norm formation in social influence networks", Social Network, (23), 167-189.

Friedkin, Noah E. y Eugene. C. Johnsen (2001). "Social influence network and opinion change". Social Network. 19, 209-222.

Grossetti, Michel (2008). "Réseaux sociaux et ressources de médiation dans l'activité économique“, Sciences de la société, 73, 83-103.

Grossetti, Michel (2004). Sociologie de l'imprévisible. Dynamique de l'activité et des formes sociales, París: Presses universitaires de France.

Kalish, Yuval y Garry Robins (2006). "Psychological predispositions and network structure: The relationship between individual predispositions, structural holes and network closure", Social Networks, 28, 56-84.

McPherson, Miller, Smith-Lovin, Lynn, y James M. Cook (2001). "Birds of a feather. homophily in social networks", Annual review of sociology, 27, 415-444.

Rojas, Fabio y Tom Howe (2004). Structure matters: an analysis of consensus formation in social networks, Comunicación presentada en la American sociological association conference, San Francisco.

Rudd, Peter y Karen Evans (1998). "Structure and agency in youth transitions: student experiences of vocational further education", J ournal of Youth Studies, 1 (1), 39-62. 
REDES- Revista hispana para el análisis de redes sociales

Vol.16,\#6, Junio 2009

http: // revista-redes. rediris.es

Steglich, Christian, Snijders, Tom A. B., y Michael Pearson (2007). Dynamic networks and behavior: separating selection from influence, Groningen: Interuniversity center for social science, theory and methodology.

Wyn, Johanna, y Peter Dwyer (1999). "New directions in research on youth in transition", J ournal of Youth Studies, 2(1), 5-21. 